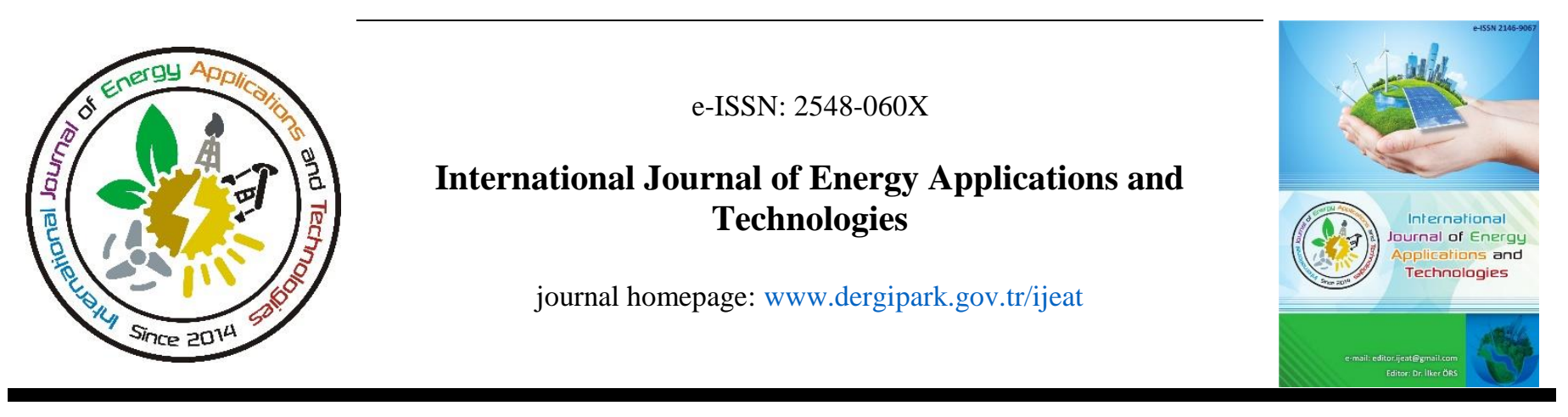

Original Research Article

\title{
Kinetics and thermodynamics of lipids extraction from microalgae using $n$-hexane
}

\author{
Satyam Parida, Satya M. Biswal* \\ Department of Biotechnology, North Odisha University of Agriculture \& Technology, Odisha, India
}

\author{
ARTICLE INFO \\ * Corresponding author \\ sbiswal@email.com \\ Received May 13, 2020 \\ Accepted July 16, 2020 \\ Published by Editorial Board \\ Members of IJEAT \\ (C) This article is distributed by \\ Turk Journal Park System under \\ the CC 4.0 terms and conditions. \\ doi: 10.31593/ijeat.734640
}

\begin{abstract}
The growth kinetics and bioenergetics of microalgae is well studied; however, the kinetics and thermodynamics of extraction of lipids from microalgae is poorly understood. The present study focuses on the kinetics and thermodynamics of the lipid extraction process from microalgae Chlamydomonas reinhardtii using n-hexanein a Soxhlet extractor. The extraction process was shown to increase lipids extraction with temperature (from 35 to $55^{\circ} \mathrm{C}$ ). Further, at a given temperature, the percent lipid extraction is linearly increased till 1.5 hours and then remained almost constant. The statistical analysis including parameters like correlation coefficient $\left(R^{2}\right)$, the root mean square $(R M S)$, standard deviation $(S D)$ and standard error of estimation (SEE) were used to establish the relevance of each model. The series of best kinetic models from high to low prominence is pseudo-second order, hyperbolic, Elovich's, parabolic and power model. The enthalpy and entropy of the present system is $266.31 \mathrm{~kJ} / \mathrm{mol}$ and $0.924 \mathrm{~kJ} / \mathrm{mol}-\mathrm{K}$, respectively. The Gibb's free energy decreased from -19.053 to $-37.412 \mathrm{~kJ} / \mathrm{mol}$ as the extraction temperature increased. The kinetic and thermodynamics parameters evaluation suggested that the microalgal lipid extraction using $n$-hexane is efficient and a spontaneous process.
\end{abstract}

Keywords: Microalgae; Lipids; Extraction; Solvent; Non-linear models

\section{Introduction}

Biofuel has been a topic of research and demand as an alternate current source of renewable form of energies. Many crops like soybean, corn, and sunflower oil have been studied extensively, from their production to their marketing; however, these crops cannot be used at an expense of food and feed support [1]. Alternatively, microalgae has shown extensive promise, but still required a lot of research in terms of making economically feasible fuel [2]. Microalgae have higher growth rates, can be grown phototorpically [3], and moreover to the best, doesn't compete for food [1, 2, 4].

Chlamydomonas reihardtiiis a single-celled green alga and of particular interest, because of its ability to contain up to $15 \%$ lipids by mass when grown under phototrophic conditions [5, 6]. In literature, there have been a lot of studies on its growth kinetics, and it's improvement on maximizing the biomass with maximum lipids [6-11]. Further, it's kinetic modeling and bioenergetics studies have been published [3, 8, 9, 11-13]. However, the kinetics and thermodynamics of extraction of oil from microalgae is not well studied.

The lab and pilot scales suggested that the growth and lipid can be monitored by regulating the media contents especially nitrogen to increase both growth and lipid contents $[3,5,6]$. Reports have suggested that lipids can be increased by stresses like nitrogen deprivation [3, 5, 6, 8, 10] or temperature [9, 12]. Microalgae can be continuously produced for both biomass and lipids simultaneously [6]. Moreover, the temperature has shown significant effects on growth and lipid concentration $[9,12]$. The energy extensive processes like removal of water from microalgae, and extraction of lipids from dried or wet microalgae are the big 
hurdles in on-going microalgal research $[2,14]$. This study stretches on the kinetics and thermodynamics of extraction of microalgal lipid from solvent n-hexane.

The lipid extraction thermodynamics and kinetics can play a crucial role in understanding the importance to decide usage of the method industrially. Presently, there is little information in literature talk about suitability of any extraction process for lipid from microalgae. The present study focuses on lipid extraction from microalgae $C$. reinhardtii using n-hexane, and the experimental data was theoretically fitted to five kinetic models: parabolic, power law, hyperbolic, Elovich's and pseudo-second order (Table 1) to understand the process parameters related to kinetic process. The statistical analysis established the relevance of each model. The correlation coefficient $\left(R^{2}\right)$, the root mean square $(R M S)$, standard deviation $(S D)$ and standard error of estimation (SEE) were determined. Finally, the thermodynamic parameters like entropy, enthalpy and Gibbs free energy parameters were estimated to test the feasibility of the process for the extraction of microalgal lipids.

\section{Materials and Method}

\subsection{Microalgae growth}

Chlamydomonas reinhardtii was grown phototrophically in minimal media [3]. A bubble based airlift photobioreactor was used with an airflow rate of $0.5 \mathrm{~L}$ of air $/ \mathrm{L}$ of media and continuous photoirradiation of $500 \mu \mathrm{mol}$ photons $\mathrm{m}^{-2} \mathrm{~s}^{-1}$. To increase both mass and lipids, first microalgae was cultured in high nitrogen rich media for 8 days and then transferred to nitrogen-deprived media for next three days [3]. The dry weight of microalgae reached $8-10 \mathrm{~g} / \mathrm{L}$. The microalgae was separated from water using centrifugation at $1000 \mathrm{xg}(\mathrm{rpm})$ for 10 minutes. Finally, the microalgae was dried at $70^{\circ} \mathrm{C}$ oven till the dry weight was constant.

\subsection{Microalgae lipid extraction}

A Soxhlet extractor was used for the extraction in which 20 $\mathrm{g}$ of dry microalgae was mixed with $200 \mathrm{ml}$ of $\mathrm{n}$-hexane. The microalgae and solvent ratio was kept constant in all experiments to 1:10. In each cycle, n-hexane with lipids passes through the pores of Soxhlet thimble that filled the syphon tube and finally reaches the $250 \mathrm{ml}$ round bottom flask. A series of experiments were designed where the Soxhlet extractor temperature was kept at $35,40,45,50$, and $55{ }^{\circ} \mathrm{C}$. The samples were collected at different interval of time and the residual hexane was evaporated by heat the mix at $65{ }^{\circ} \mathrm{C}$. The lipid yield was calculated as:

$\%$ lipid yield $=\frac{\text { weight of lipid extracted }(g)}{\text { dryweight of microalgae }(g)}$

\subsection{Non-linear kinetic models}

A total of five models were opted to fit the experimental data and compared to correlate the percent lipid yield $(\bar{q})$ with time $(t)$. The non-linear equations for these five models are:

2.3.1. Parabolic diffusion: $\bar{q}=A_{0}+A_{1} t^{1 / 2}$

Where $A_{0}$ is washing coefficient (dimensionless parabolic diffusion parameter) and $A_{1}$ is the diffusion rate constant $\left(h^{-0.5}\right)$.

\subsubsection{Power law: $\bar{q}=B t^{n}$}

Where $B$ is power law parameter that incorporates the extraction system characteristics $\left(\mathrm{h}^{-\mathrm{n}}\right)$ and $n$ is diffusional power law parameter (dimensionless power law parameter).

2.3.3. Hyperbolic: $\bar{q}=\frac{C_{1} t}{1+C_{2} t}$

Where $C_{l}$ is extraction rate at the $t=0\left(\mathrm{~h}^{-1}\right)$ and $C_{2}$ is constant defining the maximum percent lipid yield $\left(\mathrm{h}^{-1}\right)$.

2.3.4. Elovich's model: $\bar{q}=E_{0}+E_{1} \ln (t)$

Where $E_{0}$ and $E_{l}$ are Elovich's model parameter describing lipid extraction efficiency.

2.3.5. Pseudo second-order: $\frac{t}{\bar{q}}=\frac{t}{C_{s}}+\frac{1}{k C_{s}^{2}}$

Where $C s$ is equilibrium concentration of lipid in the solvent after extraction process $(\mathrm{g} / \mathrm{L})$ and $k$ is extraction rate constant.

\subsection{Statistical method}

The best fitting of the models was established by the statistical correlations. The parameters that were estimated were the coefficient of determination-correlation coefficient $\left(R^{2}\right)$, the root mean square $(R M S)$, standard deviation $(S D)$ and standard error of estimation (SEE). A high value $R^{2}$ and the low value of $R M S, S E E$ and $S D$ represents a better fitting of five models used in the study.

The RMS, SEE, and $S D$ were calculated using the following expressions:

$$
\begin{aligned}
& R M S=\sqrt{\frac{1}{N} \sum_{i=1}^{N}\left(\frac{q_{\text {exp }}-q_{\text {cal }}}{q_{\text {exp }}}\right)^{2}} \\
& S E E=\sqrt{\frac{\sum(x-y)^{2}}{d t}} \\
& S D=\sqrt{\frac{1}{N-1} \sum_{i=1}^{N}\left(\frac{q_{\text {exp }}-q_{c a l}}{q_{\text {exp }}}-\left(\frac{1}{N} \sum_{i=1}^{N} \frac{q_{\text {exp }}-q_{\text {cal }}}{q_{\text {exp }}}\right)\right)^{2}}
\end{aligned}
$$

\subsection{Thermodynamics parameters}

The Gibbs free energy $(\Delta G, \mathrm{~kJ} / \mathrm{mol})$, enthalpy $(\Delta H, \mathrm{~kJ} / \mathrm{mol})$ and entropy $(\triangle S, \mathrm{~kJ} / \mathrm{mol}-\mathrm{K})$ were calculated using following equations:

$$
\begin{aligned}
& K=\frac{Y_{T}}{Y_{U}} \\
& \Delta G=\Delta H-T \Delta S \\
& \Delta G=-R T \ln K
\end{aligned}
$$

Equating equations (6) and (7), the expression for relating $\mathrm{K}$ and $\mathrm{T}$ can be expressed in linear form of 


$$
\ln K=-\left(\frac{\Delta H}{R}\right) \frac{1}{T}+\frac{\Delta S}{R}
$$

Here, $K$ is the equilibrium constant, $Y_{T}$ is lipid yield at temperature $T, Y_{U}$ is lipid remaining in microalgae after extraction at temperature $T$, and $R$ is gas constant $(\mathrm{J} / \mathrm{mol}-\mathrm{K})$.

\section{Results and Discussion}

\subsection{Effect of temperature on lipid extraction}

Figure 1 presents results on the percent lipid yield extracted using n-hexane as the solvent at temperature $35,40,45,50$, and $55^{\circ} \mathrm{C}$. The lipids were extracted every half hour till 2.5 hours.

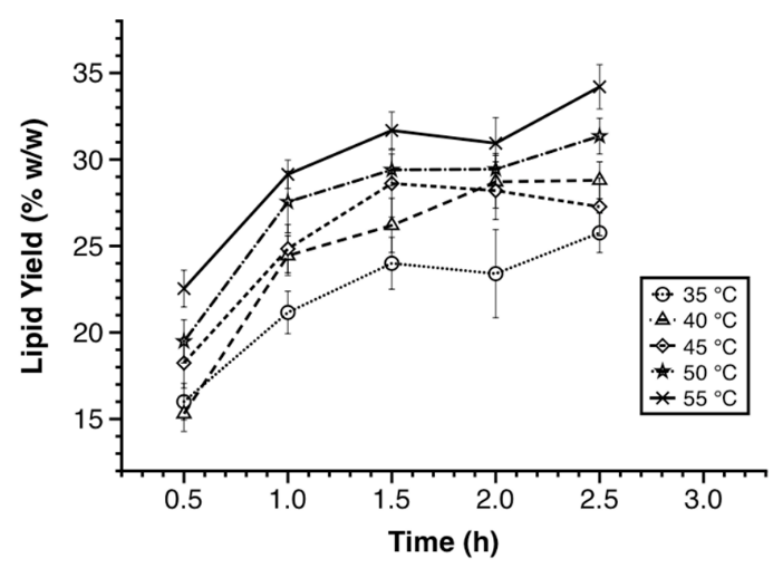

Fig. 1. Effect of temperature on microalgae lipid extraction with $n$ hexane

Each experimental reading is average of three different sets of samples. It was expected that temperature would increase the extraction process, as the heat will allow the solvent to diffuse deep in the microalgae to recover the total lipids. Further, with increase in temperature, the mass transfer rates of solvent and lipid increase significantly and thus attributing to increase in lipid with raise in extraction temperature. As it can be seen in the Figure 1, the lipid amount was increased from 15.3 to $25.76 \%$ at $35{ }^{\circ} \mathrm{C}, 16.1$ to $27.3 \%$ at $40{ }^{\circ} \mathrm{C}, 18.24$ to $28.8 \%$ at $45{ }^{\circ} \mathrm{C}, 19.49$ to $31.35 \%$ at $50{ }^{\circ} \mathrm{C}$, and 22.54 to $34.2 \%$ at $55{ }^{\circ} \mathrm{C}$ extraction temperature. The kinetics of lipid extraction with time suggested a linear relation till 1.5 hours and then the lipid extraction becomes saturated, suggesting 1.5 hours of extraction time is sufficient. A similar pattern of oil extraction was observed in Castor [15], Jatropha [16, 17], Linn [18], Olive [19], Coconut oil [20] and Sterculiasoetida seeds [21]. Similarly, in other microalgae, a similar effect of temperature can be seen $[2,14,22]$.

\subsection{Comparative fitness of kinetic models on experimental data}

The kinetic parameter obtained after fitting five kinetic models, namely, parabolic, power, hyperbolic, Elovich's and pseudo second-order models were investigated and these parameters are listed in Table 1.
Table 1. Best-fit parameters of five models used to fit the experimental lipid extraction from microalgae $\left[A_{0}\right.$ is washing coefficient (dimensionless parabolic diffusion parameter); $A_{1}$ is the diffusion rate constant $\left(\mathrm{h}^{-0.5}\right) ; B$ is power law parameter that incorporates the extraction system characteristics $\left(\mathrm{h}^{-\mathrm{n}}\right) ; n$ is diffusional power law parameter (dimensionless power law parameter); $C_{l}$ is extraction rate at the $t=0\left(\mathrm{~h}^{-1}\right) ; C_{2}$ is constant defining the maximum percent lipid yield $\left(\mathrm{h}^{-1}\right) ; E_{0}$ and $E_{l}$ are Elovich's model parameter describing lipid extraction efficiency; $C s$ is equilibrium concentration of lipid in the solvent after extraction process $(\mathrm{g} / \mathrm{L}) ; k$ is extraction rate constant.]

\begin{tabular}{|c|c|c|c|c|c|}
\hline Parameter & $35^{\circ} \mathrm{C}$ & $40^{\circ} \mathrm{C}$ & $45^{\circ} \mathrm{C}$ & $50^{\circ} \mathrm{C}$ & $55^{\circ} \mathrm{C}$ \\
\hline \multicolumn{6}{|l|}{ 1. Parabolic } \\
\hline A0 & 6.7536 & 7.5211 & 9.1974 & 12.0748 & 13.6132 \\
\hline A1 & 1.8845 & 1.9078 & 1.9157 & 1.9032 & 1.7937 \\
\hline $\mathrm{R}^{2}$ & 0.9380 & 0.9213 & 0.9141 & 0.9192 & 0.9101 \\
\hline RMS & 0.0525 & 0.0574 & 0.0515 & 0.0462 & 0.0487 \\
\hline SEE & 0.4414 & 0.5330 & 0.4961 & 0.4867 & 0.5523 \\
\hline SD & 0.0129 & 0.0143 & 0.0120 & 0.0093 & 0.0093 \\
\hline \multicolumn{6}{|l|}{ 2. Power } \\
\hline B & 14.2304 & 16.1469 & 17.1500 & 18.5501 & 21.1704 \\
\hline $\mathrm{n}$ & 0.1019 & 0.1023 & 0.0976 & 0.1031 & 0.0972 \\
\hline $\mathrm{R}^{2}$ & 0.8774 & 0.8671 & 0.8660 & 0.8566 & 0.8418 \\
\hline RMS (\%) & 0.1703 & 0.1727 & 0.1650 & 0.1635 & 0.1818 \\
\hline SEE & 1.3600 & 1.3129 & 1.3977 & 1.5362 & 1.7981 \\
\hline SD & 0.0135 & 0.1110 & 0.2288 & 0.2985 & 0.3748 \\
\hline \multicolumn{6}{|c|}{ 3. Hyperbole } \\
\hline $\mathrm{C} 1$ & 0.8694 & 0.9572 & 1.2157 & 1.4449 & 1.5829 \\
\hline $\mathrm{C} 2$ & 0.0241 & 0.0254 & 0.0299 & 0.0348 & 0.0354 \\
\hline $\mathrm{R}^{2}$ & 0.9824 & 0.9783 & 0.9802 & 0.9818 & 0.9855 \\
\hline RMS (\%) & 0.0210 & 0.0270 & 0.2003 & 0.0205 & 0.0185 \\
\hline SEE & 0.2141 & 0.2933 & 0.2205 & 0.2370 & 0.2532 \\
\hline SD & 0.0012 & 0.0002 & 0.0006 & 0.0011 & 0.0049 \\
\hline \multicolumn{6}{|l|}{ 4. Elovich } \\
\hline E0 & 11.2416 & -10.9847 & -8.7219 & -6.4403 & -4.2827 \\
\hline E1 & 7.7110 & 8.4875 & 8.0718 & 7.7516 & 8.2526 \\
\hline $\mathrm{R}^{2}$ & 0.9742 & 0.9694 & 0.9737 & 0.9657 & 0.9587 \\
\hline RMS & 0.0307 & 0.0354 & 0.0308 & 0.0308 & 0.0289 \\
\hline SEE & 0.2537 & 0.3430 & 0.3148 & 0.3373 & 0.3621 \\
\hline SD & 0.0054 & 0.0070 & 0.0049 & 0.0433 & 0.0042 \\
\hline \multicolumn{6}{|c|}{ 5. Second Order } \\
\hline Cs & 33.6934 & 35.8111 & 37.2150 & 39.4571 & 42.0519 \\
\hline $\mathrm{K}$ & 7.36E-04 & $6.81 \mathrm{E}-04$ & $7.51 \mathrm{E}-04$ & 8.01E-04 & $9.07 \mathrm{E}-04$ \\
\hline $\mathrm{R}^{2}$ & 0.9939 & 0.9868 & 0.9897 & 0.9906 & 0.9501 \\
\hline RMS & 0.2144 & 0.0271 & 0.0205 & 0.0187 & 0.0183 \\
\hline SEE & 0.1888 & 0.2816 & 0.2148 & 0.2361 & 0.2387 \\
\hline SD & 0.0008 & 0.0030 & 0.0005 & 0.0047 & 0.0011 \\
\hline
\end{tabular}


The parameters of all the studied models were appeared to increase with increase in temperature. The solvent extraction of oil from various seeds has shown a similar trend in the close agreement with this study.

The fitness of each model was statistically determined and the values of $R^{2}, R M S, S E E$ and $S D$ given in equations (2)-(4) were the determining factors to keep or avoid a model for the present study experimental data analysis. The best fit would have a higher value of $R^{2}$ and lower values of $R M S, S E E$, and $S D$. The best fit-curves show all models fitted well as shown in Figure 2. The second order model had the highest $R^{2}$ ranging from 0.95 to 0.99 and lowest values of $R M S$ ranging from 0.0183 to 0.0271 , SEE ranging from 0.1888 to 0.2816 , and $S D$ ranging from 0.0005 to 0.0047 . Power law showed the worst fitness with $R^{2}$ value ranging from 0.8418 to 0.8774 , and higher values of $R M S, S E E$, and $S D$ compared to the other models (RMS ranged from 0.1635 to $0.188, S E E$ ranged from 1.7981 to 1.3192 , and $S D$ ranged from 0.0135 to 0.3748). In the order of best to worst, the order of models was: Pseudo-second order, hyperbolic, Elovich's, parabolic and power model. Clearly, pseudo-second order kinetic model was best to describe the lipid extraction using solvent from microalgae.
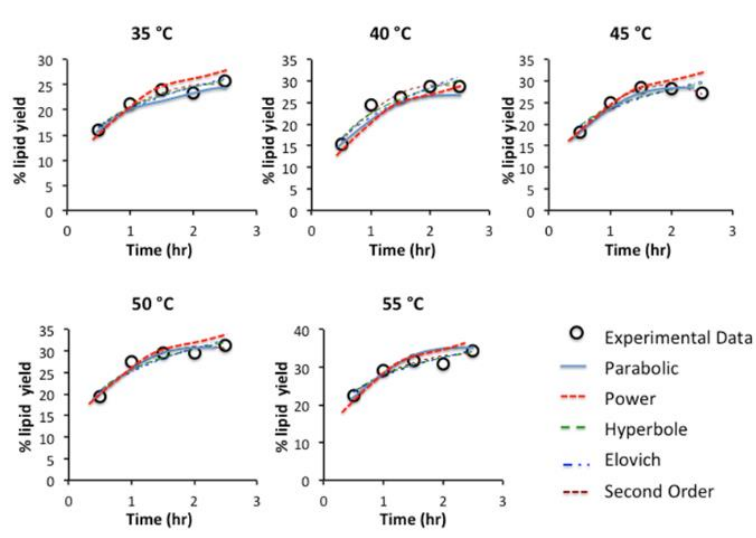

Fig. 2. Best-fit curves for the models used to fit the experimental data for lipid extraction from microalgae

\subsection{Thermodynamic parameters estimation and relevance}

The thermodynamic parameters were estimated by first calculating $K$ values at different temperatures using Eq. (5) (Table 2). Then, $\ln K$ and $1 / T$ were fitted to calculate the values of $\Delta G, \Delta H$, and $\Delta S$ using Eq (6) and (7). The best fit suggested linearity between $\ln K$ and $1 / T$ with $R^{2}$ value of 0.95 (Fig. 3).The enthalpy and entropy of the present study extraction system is $266.31 \mathrm{~kJ} / \mathrm{mol}$ and $0.924 \mathrm{~kJ} / \mathrm{mol}-\mathrm{K}$, respectively. It has been reported for oil extraction from olive cakes to $182.81-598.74 \mathrm{~kJ} / \mathrm{mol}$ and from coconut ranged from $4-13.5 \mathrm{~kJ} / \mathrm{mol}$.

These results indicate that the extraction process was endothermic and external energy sources to extract the lipids from microalgae. The positive values of entropy change for the entire process were an indication that the process was irreversible. Further, the irreversibility of this extraction process at all studied temperatures was confirmed in terms of positive entropy change. The free energy of the extraction process decreases with increasing temperature suggesting 55 ${ }^{\circ} \mathrm{C}$ as the best extraction temperature. The Gibb's free energy decreased from -19.053 to $-37.412 \mathrm{~kJ} / \mathrm{mol}$ as the extraction temperature increased from 35 to $55^{\circ} \mathrm{C}$. The higher values of free energy suggested that the process were more spontaneous with increase in temperature, which can be attributed to better diffusion and mass transfer of solvent in microalgae.

Table 2. The values of $\mathrm{K}$ and $\mathrm{G}$ estimated for the lipid extraction process at different temperatures from microalgae using n-hexane

\begin{tabular}{lccc}
\hline $\boldsymbol{T}$ & $\boldsymbol{T}$ & $\boldsymbol{K}$ & $\boldsymbol{G}$ \\
${ }^{\circ} \mathbf{C}$ & ${ }^{\circ} \mathbf{K}$ & & $\mathbf{k J} / \mathbf{m o l}$ \\
\hline 35 & 308.15 & 3704.4 & -19.053 \\
40 & 313.15 & 7129.5 & -22.4749 \\
45 & 318.15 & 57424 & -28.469 \\
50 & 323.15 & 693720 & -35.2013 \\
55 & 328.15 & 1019200 & -37.4121 \\
\hline
\end{tabular}

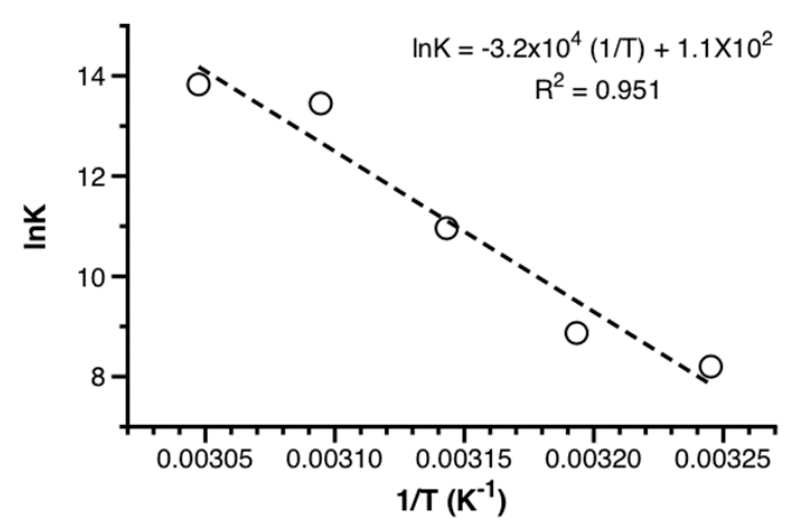

Fig. 3. Plot between $\ln K$ and $1 / T$ to estimate the thermodynamics parameters

\section{Conclusions}

The kinetics and thermodynamics for n-hexane based extraction of lipid from microalgae Chlamydomonas reinhardtii was achieved. Lipid percent was found to be both time and extraction temperature dependent. Within studied temperature range $\left(35\right.$ to $\left.55{ }^{\circ} \mathrm{C}\right)$, the values of percent lipid increase with the increase in temperature. However, at a given temperature, the percent lipid increased linearly with time till 1.5 hours and then saturated. So, extraction time of 1.5 hours and extraction temperature of $55^{\circ} \mathrm{C}$ provided the maximum lipids extraction. More, the experimental data was fitted for five kinetic models in which pseudo-second order 
was the best and power based fitting presented the least accurate of five models. The thermodynamic study revealed the enthalpy, entropy and free energy of the studied extraction system suggesting that Soxhlet extraction of microalgal lipids is well suited for extracting lipids from microalgae.

\section{$\underline{\text { ORCID }}$}

Satyam Parida

0000-0002-0623-3345

Satya M. Biswal

0000-0003-4875-607X

\section{References}

[1] Tenenbaum D.J. 2008.Food vs. fuel: diversion of crops could cause more hunger.Environ. Health Persp.,116, A254-A257.

[2] Derakhshan M.V., Nasernejad B., Abbaspour-Aghdam F., Hamidi M.2015.Oil extraction from algae: A comparative approach. Biotechnol. Appl. Biochem., 62,375-382.

[3] Tevatia R., Demirel Y., Blum P. 2012.Kinetic modeling of photoautotropic growth and neutral lipid accumulation in terms of ammonium concentration in Chlamydomonasreinhardtii.Bioresour. Technol., 119, 419-424.

[4] Canakci M. and Sanli H.2008. Biodiesel production from various feedstocks and their effects on the fuel properties,J. Ind. Microbiol.Biotechnol., 35, 431-441.

[5] James G.O., Hocart C.H., Hillier W., Chen H., Kordbacheh F., Price G.D., Djordjevic M.A. 2011.Fatty acid profiling of Chlamydomonasreinhardtii under nitrogen deprivation,Bioresour.Technol., 102, 33433351.

[6] Tevatia R., Allen J., Blum P., Demirel Y., Black P.2014 Modeling of rhythmic behavior in neutral lipid production due to continuous supply of limited nitrogen: Mutual growth and lipid accumulation in microalgae Bioresour. Technol., 170, 152-159.

[7] Scranton M.A., Ostrand J.T., Fields F.J., Mayfield S.P.2015.Chlamydomonas as a model for biofuels and bio-products production Plant J., 82, 523-531.

[8] Siaut M., Cuiné S., Cagnon C., Fessler B., Nguyen M., Carrier P., Beyly A., Beisson F., Triantaphylides C., LiBeisson Y., Peltier G. 2011.Oil accumulation in the model green alga Chlamydomonasreinhardtii: characterization, variability between common laboratory strains and relationship with starch reserves. BMC Biotechnol., 11, 7.

[9] Tevatia R., Demirel Y., Rudrappa D., Blum P.2015. Effects of thermodynamically coupled reaction diffusion in microalgae growth and lipid accumulation: Model development and stability analysis. Comput. Chem. Eng., 75, 28-39.
[10] Yang L., Chen J., Qin S., Zeng M., Jiang J., Hu L., Xiao P., Hao W., Hu, Z., Lei A., Wang J. 2018.Growth and lipid accumulation by different nutrients in the microalga Chlamydomonasreinhardtii.Biotechnol. Biofuels., 11, 40.

[11] Zhang X.W., Chen F., Johns M.R.1999. Kinetic models for heterotrophic growth of Chlamydomonasreinhardtii in batch and fed-batch cultures.Process Biochem., 35, 385-389.

[12] Allen J.W., Tevatia R., Demirel Y., DiRusso C.C., Black P.N. 2018Induction of oil accumulation by heat stress is metabolically distinct from $\mathrm{N}$ stress in the green microalgaeCoccomyxasubellipsoidea C169. PloS One, 13, e0204505-e0204505.

[13] Küçük K., Tevatia R., Sorgüven E., Demirel Y., Özilgen M. 2015Bioenergetics of growth and lipid production in Chlamydomonasreinhardtii. Energy, 83, 503-510.

[14] Cooney M., Young G., Nagle N. 2009.Extraction of Bio-oils from Microalgae.Sep. Purif. Rev., 38, 291-325.

[15] Abdelmoez W., Ashour E., Naguib S.M., Hilal A., Al Mahdy D.A., Mahrous E.A., Sattar E.A. 2016.Kinetic and Thermodynamics studies for Castor Oil Extraction Using Subcritical Water Technology.J. Oleo Sci., 65, 477-485.

[16] Devappa R.K., Makkar H.P.S., Becker K. 2010.Optimization of conditions for the extraction of phorbol esters from Jatropha oil.Biomass Bioenergy, 34, 1125-1133.

[17] SantosS.B.d., Martins M.A., Caneschi A.L., Aguilar P.R.M., J. S. R. Coimbra J.S.R. 2015. Kinetics and Thermodynamics of Oil Extraction from Jatrophacurcas L. Using Ethanol as a Solvent.Int. J. Chem. Eng., 2015, 9.

[18] Mathiarasi R. and N. Partha N.2016.Optimization, kinetics and thermodynamic studies on oil extraction from Daturametel Linn oil seed for biodiesel production,Renew. Energy., 96, 583-590.

[19] Meziane S., Kadi H. 2008.Kinetics and Thermodynamics of Oil Extraction from Olive Cake. $J$. Am. Oil Chem. Soc., 85, 391-396.

[20] Sulaiman S., Abdul Aziz A.R., KheireddineAroua M. 2013. Optimization and modeling of extraction of solid coconut waste oil. J. Food Eng., 114, 228-234.

[21] Sivakumar P.,Parthiban K.S., Sivakumar P., Vinoba M., Renganathan S.2012.Optimization of Extraction Process and Kinetics of Sterculiafoetida Seed Oil and Its Process Augmentation for Biodiesel Production.Ind.Eng. Chem. Res., 51, 8992-8998.

[22] Patil P.D., Dandamudi K.P.R., Wang J., Deng Q., Deng S.2018. Extraction of bio-oils from algae with supercritical carbon dioxide and co-solvents. $J$. Supercrit. Fluid, 135, 60-68. 